Open Access

\title{
Phase II trial of selective internal radiation therapy and systemic chemotherapy for
} liver-predominant metastases from pancreatic adenocarcinoma

Peter Gibbs ${ }^{1,2^{*}}$, Cuong Do², Lara Lipton ${ }^{1,2}$, David N. Cade ${ }^{3}$, Michael J. Tapner ${ }^{3}$, David Price ${ }^{4}$, Geoff D. Bower ${ }^{5}$, Richard Dowling ${ }^{6}$, Meir Lichtenstein ${ }^{7}$ and Guy A. van Hazel $^{8}$

\begin{abstract}
Background: This prospective, open-label phase II study assessed the impact of liver-directed therapy with selective internal radiation therapy (SIRT) and systemic chemotherapy on progression-free survival (PFS) in liver-dominant metastatic pancreatic adenocarcinoma.

Methods: Patients received yttrium-90-labelled $\left({ }^{90}\right.$ Y) resin microspheres (SIR-Spheres; Sirtex Medical Limited, Sydney, Australia) as a single procedure on day 2 of the first weekly cycle of 5 -fluorouracil $\left(5 \mathrm{FU} ; 600 \mathrm{mg} / \mathrm{m}^{2}\right)$ with the option to switch to gemcitabine $\left(1000 \mathrm{mg} / \mathrm{m}^{2}\right)$ after 8 weeks of 5FU. Statistical analysis was conducted using Microsoft Excel (Microsoft Corporation, Redmond, Washington, USA). The primary endpoint of the study was PFS in the liver, with a median of $\geq 16$ weeks defined as the threshold for clinical significance. PFS and overall survival (OS) were summarised by the Kaplan-Meier method using non-parametric estimates of the survivor function.

Results: Fourteen eligible patients were enrolled; ten had primary tumour in situ and eight had liver-only metastases. Patients received a median ${ }^{90} \mathrm{Y}$ activity of $1.1 \mathrm{GBq}$ and 8 weekly doses of $5 \mathrm{FU}$; seven patients received a median of two doses of gemcitabine. Disease control in the liver was $93 \%$ (two confirmed partial responses [PR], one unconfirmed PR, ten stable disease). Median reduction in cancer antigen 19-9 was $72 \%$. Median PFS was 5.2 months in the liver, which met the primary endpoint of the study, and 4.4 months at any site. PFS was prolonged in those with a resected primary compared with patients with primary in situ (median 7.8 vs. 3.4 months; $p=0.017$ ). Median OS was 5.5 months overall and 13.6 months in patients with a resected primary. Grade $3 / 4$ adverse events occurred in eight (57 \%) patients during days $0-60$. There was one sudden death and another patient who died from possible treatment-related liver failure 7.0 months after SIRT.

Conclusions: SIRT and chemotherapy appears to be an effective treatment for liver metastases from pancreatic cancer, likely to be of most benefit in selected patients with a resected primary tumour and liver only disease. Significant toxicity was observed and the safety of this approach in patients with metastatic pancreatic cancer will need to be confirmed in subsequent studies. Further study is warranted with SIRT and modern chemotherapies.
\end{abstract}

Trial registration: ACTRN12606000015549

Keywords: Advanced, Liver, Metastases, Pancreas, Radioembolization, SIRT

\footnotetext{
* Correspondence: Peter.Gibbs@mh.org.au
'Department of Medical Oncology, Royal Melbourne Hospital, Grattan Street,

*Correspondence: Peter.Gibbs@mh.org.au
'Department of Medical Oncology, Royal Melbourne Hospital, Grattan Street, Parkville, VIC 3050, Australia

${ }^{2}$ Department of Medical Oncology, Western Hospital, Melbourne, Australia

Full list of author information is available at the end of the article
}

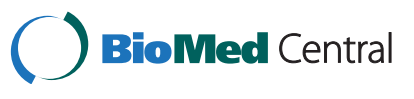

(c) 2015 Gibbs et al. Open Access This article is distributed under the terms of the Creative Commons Attribution 4.0 International License (http://creativecommons.org/licenses/by/4.0/), which permits unrestricted use, distribution, and reproduction in any medium, provided you give appropriate credit to the original author(s) and the source, provide a link to the Creative Commons license, and indicate if changes were made. The Creative Commons Public Domain Dedication waiver (http://creativecommons.org/publicdomain/zero/1.0/) applies to the data made available in this article, unless otherwise stated. 


\section{Background}

Pancreatic cancer is the fourth leading cause of cancerrelated deaths in the USA and the fifth leading cause of cancer deaths in Europe with the incidence continuing to rise $[1,2]$. The majority of patients have locally advanced and/or metastatic disease at presentation, resulting in a dismal 5-year survival rate of less than $5 \%$ [3]. At the time that this study was conducted, gemcitabine was the most widely used systemic treatment for metastatic pancreatic cancer. In a phase III study, gemcitabine had a modest survival benefit of 5.6 months compared with 4.4 months for 5 -fluorouracil (5FU) [4], and so the rationale for treatment with gemcitabine was primarily the alleviation of diseaserelated symptoms rather than extending overall survival (OS) [4]. Since then, the landmark European PRODIGE-4 trial with combined chemotherapy with folinic acid, fluorouracil, irinotecan and oxaliplatin (FOLFIRINOX) has extended the OS of patients with metastatic pancreatic cancer beyond 10 months (11.1 months vs. 6.8 months with gemcitabine) [5]. However, despite improvements in the control of locally advanced and metastatic disease with FOLFIRINOX [5-7], a recent published multicentre evaluation showed that Zapproximately one-third of patients were hospitalised due to adverse events [8] and it remains uncertain how widely this regimen will be used in the routine management of pancreatic cancer.

Liver metastases are a dominant cause of treatment failure in pancreatic cancer, occurring in $25-53 \%$ of patients even after loco-regional control with chemo-radiation and surgical resection [9]. For patients with advanced disease, the PRODIGE-4 trial showed hepatic metastases and declining liver function (defined by albumin levels $<3.5 \mathrm{~g} / \mathrm{dL}$ ) remain independent adverse prognostic factors for OS [5]. Consequently, liver-directed therapies, which improve disease control in the liver, may be of value in extending OS when combined with systemic chemotherapy.

Selective internal radiation therapy (SIRT) with yttrium$90\left({ }^{90} \mathrm{Y}\right)$-labelled microspheres is a loco-regional treatment, which has been evaluated in patients with hepatocellular carcinoma [10-12], as well as liver-dominant neuroendocrine tumours [13, 14], colorectal cancer (CRC) [15-18] and breast cancer [19-21]. To date, however, there have been very limited published data on SIRT in pancreatic cancer [22, 23]. Following the positive experience with SIRT in these primary and secondary hepatic neoplasms, we conducted a prospective study to assess whether SIRT, combined with 5FU, would extend PFS in the liver, and consequently OS, in patients with liver-only or liverdominant advanced pancreatic cancer.

\section{Methods}

\section{Study design}

This was a prospective, open-label, multicentre, phase II trial to assess the safety and efficacy of SIRT combined with
5FU in patients with recently diagnosed liver-dominant metastases from pancreatic adenocarcinoma. Enrolled patients were to have liver metastases as the dominant clinical issue and the site of disease that threatened the patient's life. This criterion was created with the expectation that enrolled patients would have bulky liver metastases, and small volume or no extra-hepatic disease.

Patients received SIRT using ${ }^{90} Y$-resin microspheres (SIR-Spheres; Sirtex Medical Limited, Sydney, Australia) as a single procedure, 2 days after the first bolus injection of 5FU $600 \mathrm{mg} / \mathrm{m}^{2}$ (administered once weekly). Patients with on-going response could continue to receive chemotherapy for 16 weeks, until disease progression or unacceptable toxicity. At the time the protocol was written the standard treatment options for metastatic pancreatic cancer were gemcitabine and 5FU. Given the safety data for the combination of 5FU and SIRT and in order to avoid the well-documented radiosensitising effects of gemcitabine [24, 25] initial therapy was with $5 \mathrm{FU}$ alone, Investigators had the option after 8 weeks of 5FU to switch patients to gemcitabine $1000 \mathrm{mg} / \mathrm{m}^{2}$ (given weekly for 7 weeks followed by a 1-week rest, and thereafter weekly for 3 weeks, every 4 weeks) provided that the patient had not progressed on or experienced unacceptable toxicity from gemcitabine previously.

The principal aim of the study was to evaluate hepatic disease control, using PFS in the liver as the primary endpoint. The combination of SIRT and chemotherapy would be considered to be of clinical significance if the median PFS in the liver was $\geq 16$ weeks. This was based on data from a study of $5 \mathrm{FU}$ alone, where more than $70 \%$ of patients had disease progression by 2 months [4], suggesting that if median PFS in the liver was more than twice this it would suggest significant impact from the addition of SIRT. The secondary endpoints were: safety and toxicity, PFS at any site, best objective response rate in the liver and at any site, site of disease progression and OS.

The study conformed to the World Medical Association Declaration of Helsinki and the Australian National Health and Medical Research Council statement on human experimentation. Prior approval of the study protocol was received from each institute's Human Research Ethics Committee (Melbourne Health Ethics Committee study 2006.124 and Mount Hospital Ethics Committee study EC35.3).

\section{Patients}

Patients were enrolled at two centres (Mount Medical Centre, Perth, Australia; Western Hospital, Melbourne, Australia) between October 2006 and November 2009. All patients were fully informed of the nature of the trial and signed an informed consent document. 
Patients were included in the study if they were 18 years of age or older, with a life expectancy of $\geq 2$ months without any active treatment, had a World Health Organization (WHO) performance status of 0 or 1 , and a diagnosis of pancreatic adenocarcinoma. At the time of inclusion, the liver had to be the dominant site of disease, impacting on patients' health-related quality of life and/or survival; lowvolume extra-hepatic metastases and/or an intact primary cancer were permitted. Previous chemotherapy, either as adjuvant treatment or first-line therapy for metastatic disease was permitted. All laboratory parameters had to be within the defined limits for the safe delivery of SIRT, i.e. neutrophil count $>1.5 \times 10^{9} / \mathrm{L}$; platelets $>100 \times 10^{9} / \mathrm{L}$; creatinine $<150 \mu \mathrm{mol} / \mathrm{L}$; bilirubin $\leq 1 \times$ upper limit of normal (ULN); albumin $\geq 3 \mathrm{~g} / \mathrm{dL}$. Female patients were either postmenopausal, sterile, or if sexually active using an acceptable method of contraception. Male patients, if sexually active (and not surgically sterile) and having a pre-menopausal partner, were required to use an acceptable method of contraception.

Patients were excluded with evidence of ascites, cirrhosis or portal hypertension (as determined by clinical or radiological assessment), occlusion of the main portal vein, central nervous system metastases, prior radiotherapy that included the liver in the treatment field, prior treatment with an investigational agent within 30 days of SIRT, or evidence of any concurrent condition that, in the opinion of the investigator, would render the patient ineligible for treatment according to this protocol.

\section{Assessment and data handling}

All baseline assessments were carried out within 29 days of enrolment, including serum cancer antigen 19-9 (CA19-9), and computed tomography (CT) or magnetic resonance imaging (MRI) to assess the extent of disease in the abdomen and chest. The percentage tumour burden within the liver was determined using the baseline CT/MRI scan, utilising validated tumour volumetry software (MeVis Distant Services, Bremen, Germany). Patients underwent a baseline hepatic angiogram to map the vascular anatomy of the liver. Technetium-99 $\mathrm{m}$ macroaggregated albumin was used as a surrogate for ${ }^{90}$ Y-resin microspheres during the pre-treatment planning to determine the presence and magnitude of arterio-venous shunting to the lungs so that the lung radiation exposure could be kept within safe limits $(<25 \mathrm{~Gy})$ and the activity of ${ }^{90} \mathrm{Y}$ administered was adjusted accordingly [25]. The calculated activity of ${ }^{90} \mathrm{Y}$ to be implanted was determined from tables provided by the manufacturer, based on a modification of the Body Surface Area formula and adjusted for the extent of lung shunting for each patient (see Additional file 1).

Post-SIRT, patients were evaluated every 4 weeks and tumour response was assessed every 8 weeks until disease progression in the liver according to Response
Evaluation Criteria In Solid Tumours (RECIST) version 1.0. Complete response or partial response (PR) of liver metastases were confirmed by a further CT scan performed after 4 weeks. Patients were analysed according to the presence or absence of the primary tumour in situ and the presence or absence of extra-hepatic metastases.

PFS in the liver was defined as the interval between trial entry and the date of tumour progression (based on RECIST) or death, whichever occurred sooner. OS was defined as the interval between enrolment and the date of death.

Adverse events were recorded from consent until 30 days after the last dose of protocol chemotherapy was administered. At the time of their occurrence, the causal relationship between the adverse events and the protocol therapy was recorded by the investigator. All adverse events were graded using the National Cancer Institute Common Terminology Criteria for Adverse Events (NCI-CTCAE) version 3.0. Adverse events are presented according to time of occurrence: from enrolment up to day 60 or beyond day 60 .

Statistical analysis was conducted using Microsoft Excel (Microsoft Corporation, Redmond, Washington, USA). PFS and OS was summarised by the Kaplan-Meier method using nonparametric estimates of the survivor function.

\section{Results}

\section{Baseline characteristics and treatment}

Fifteen patients were enrolled and received protocol therapy. One patient was excluded from the analysis after the histopathology report confirmed retrospectively that the liver lesion was derived from a pancreatic neuroendocrine tumour. Patient and disease characteristics for the remaining 14 patients are detailed in Table 1 . Patients entered the study a median of 13 days after diagnosis of metastatic pancreatic cancer. Most patients had a good performance status, either WHO 0 (71 \%) or 1 (29\%). The primary cancer was in situ in ten patients (71\%); six of these patients had liver-only metastases, and four had extrahepatic disease/metastases (EHD). The primary tumour had been resected in four patients (two with liver-only disease; two with EHD). Three patients had received prior chemotherapy (three received gemcitabine, two 5FU, one carboplatin); all three had liver-only metastases, two with a resected primary. The tumour burden in the liver varied between 1 and $37 \%$ of the liver volume (mean $14 \%$ ), with bilobar involvement in all patients.

All patients received SIRT 2 days after the first weekly dose of 5FU. The median prescribed and administered activity of ${ }^{90} \mathrm{Y}$-resin microspheres was $1.1 \mathrm{GBq}$ (range 0.7-2.0 GBq). ${ }^{90}$ Y-resin microspheres were administered by selective injection to left and right hepatic arteries except in one patient who did not receive SIRT in the right 
Table 1 Baseline patient and disease characteristics

\begin{tabular}{|c|c|c|c|}
\hline \multicolumn{2}{|l|}{ Characteristics } & \multicolumn{2}{|l|}{ Patients } \\
\hline Gender & Male & $\begin{array}{l}\text { Number } \\
6\end{array}$ & $\begin{array}{l}\text { Percentage } \\
43 \%\end{array}$ \\
\hline & Female & 8 & $57 \%$ \\
\hline \multicolumn{2}{|l|}{ Age, years; median (range) } & \multicolumn{2}{|c|}{$62(48-76)$} \\
\hline \multirow[t]{2}{*}{ WHO performance status } & 0 & 10 & $71 \%$ \\
\hline & 1 & 4 & $29 \%$ \\
\hline \multicolumn{2}{|c|}{ Time from diagnosis of metastatic pancreas cancer to trial entry, ${ }^{a}$ days; median (range) } & \multicolumn{2}{|c|}{$13(5-434)$} \\
\hline \multirow[t]{4}{*}{ Cancer stage at diagnosis } & TX & 4 & $29 \%$ \\
\hline & T2 & 6 & $43 \%$ \\
\hline & T3 & 2 & $14 \%$ \\
\hline & T4 & 2 & $14 \%$ \\
\hline \multirow[t]{2}{*}{ Primary tumour in situ } & Yes & 10 & $71 \%$ \\
\hline & No & 4 & $29 \%$ \\
\hline \multirow[t]{5}{*}{ Metastases } & Liver only & 8 & $57 \%$ \\
\hline & Liver and lung & 2 & $14.5 \%$ \\
\hline & Liver and lymph nodes & 2 & $14.5 \%$ \\
\hline & Liver, lung and lymph nodes & 1 & $7 \%$ \\
\hline & Liver, lung, lymph nodes, soft tissue & 1 & $7 \%$ \\
\hline \multicolumn{2}{|l|}{ Number of metastatic sites; median (range) } & \multicolumn{2}{|l|}{$3(1-5)$} \\
\hline \multicolumn{2}{|l|}{ CA19-9, ${ }^{b} \mathrm{U} / \mathrm{mL}$; median (range) } & \multicolumn{2}{|c|}{$3480(33-280,000)$} \\
\hline$>$ ULN & & 13 & $93 \%$ \\
\hline \multirow[t]{2}{*}{ Prior lines of chemotherapy for metastatic disease } & 0 & 13 & $93 \%$ \\
\hline & 1 & 1 & $7 \%$ \\
\hline
\end{tabular}

${ }^{\text {a }}$ Trial entry defined as day of informed consent

${ }^{\mathrm{b}} \mathrm{N}=13$ patients with elevated CA19-9 baseline levels (ULN $37 \mathrm{U} / \mathrm{mL}$ )

lobe due to an unfavourable hepatic arterial anatomy that precluded catheter access. Median follow-up from study entry was 5.9 months (range 1.4-16.2 months). Patients received a median of 8 weekly doses of $5 \mathrm{FU}$ (median $600 \mathrm{mg} / \mathrm{m}^{2}$; range $350-638 \mathrm{mg} / \mathrm{m}^{2}$ ). Seven patients $(50 \%)$, one of whom had progressive disease, received a median of 2 doses (range 2-10) of gemcitabine (median $1000 \mathrm{mg} / \mathrm{m}^{2}$; range, $600-1000 \mathrm{mg} / \mathrm{m}^{2}$ ), an average of 3.7 months (range 2.1-8.1 months) after the start of $5 \mathrm{FU}$.

\section{Adverse events}

Adverse events are listed in Table 2A and B. The majority of adverse events occurred during the first 60 days of therapy.

Early events (days $0-60$ ): grade $3 / 4$ adverse clinical and/ or laboratory events occurred in $8(57 \%)$ patients during this period. Nine patients $(64 \%)$ had no grade 3 or higher treatment-related clinical adverse events, and there was no grade 3 or 4 abdominal pain, nausea, vomiting or diarrhoea during this period. Two patients (14\%) developed grade 3 fatigue which may have been treatment-related.
Grade 3 biochemical toxicities were observed in two patients $(14 \%)$ and significant haematological events in four patients (28\%): grade 3 neutropenia (two patients; $14 \%$ ) and grade 3 thrombocytopenia (two patients; $14 \%$ ).

Later events (after 60 days): six patients (50\%) had grade 3 or higher treatment-related clinical adverse events, four of whom had switched to gemcitabine, with biochemical toxicities reported in six patients (50\%, three on gemcitabine) and hematologic events in three patients ( $25 \%$, all on gemcitabine).

Death without documented progression occurred in two patients. The first patient died suddenly 1.5 months after study entry and within 28 days of SIRT; the patient had no adverse events greater than grade 1 severity, laboratory tests were within normal limits but CA19-9 had increased by $293 \%$ from baseline. At enrolment, this patient had a T4 primary tumour in situ with metastases to the liver, lungs and lymph nodes. The second patient, who died 7.0 months after study entry, presented with liver-only metastases following prior resection of a T2 primary cancer. The patient died from hepatic failure, considered to possibly be due to radioembolization-induced liver disease 
Table 2 Adverse events. Adverse events (by NCl-CTCAE v.3 grade) recorded up to 60 days after the start of protocol therapy, from 61 days onwards and across the whole study period $(n=14)$

\begin{tabular}{|c|c|c|c|c|c|c|}
\hline \multirow[t]{2}{*}{ Category/event } & \multicolumn{2}{|c|}{ Day 1 to $60(n=14)$} & \multicolumn{2}{|c|}{ Day 61 onwards $(n=12)$} & \multicolumn{2}{|c|}{ Day 1 to last assessment ( } \\
\hline & Grade 1-2 & Grade $\geq 3$ & Grade 1-2 & Grade $\geq 3$ & Grade 1-2 & Gra \\
\hline \multicolumn{7}{|l|}{ A. } \\
\hline \multicolumn{7}{|l|}{ Gastrointestinal } \\
\hline Nausea & 10 & 0 & 4 & 1 & 10 & 1 \\
\hline Vomiting & 7 & 0 & 1 & 0 & 8 & 0 \\
\hline Anorexia & 5 & 0 & 5 & 2 & 6 & 2 \\
\hline Diarrhoea & 4 & 0 & 1 & 1 & 4 & 1 \\
\hline Stomatitis & 1 & 0 & 2 & 0 & 2 & 0 \\
\hline Mucositis & 2 & 0 & 0 & 0 & 2 & 0 \\
\hline Constipation & 2 & 0 & 1 & 0 & 2 & 0 \\
\hline
\end{tabular}

Pain

Abdominal pain

Pain (non-abdominal)

2

Constitutional Symptoms

Fatigue

Fever

Hepatobiliary/Pancreas

Ascites

Jaundice

Liver failure

Neurology

Neuropathy

Pulmonary/Upper Respiratory

Dyspnoea

Bruising

Pneumonia

0

0

$-1$

1

0

0

$\begin{array}{ll}3 & 0 \\ 0 & \end{array}$

vascular

Pulmonary embolism

Deep vein thrombosis

6

3

2

5

0

ade $\geq 3$


Table 2 Adverse events. Adverse events (by NCI-CTCAE v.3 grade) recorded up to 60 days after the start of protocol therapy, from 61 days onwards and across the whole study period $(n=14)$ (Continued)

\begin{tabular}{|c|c|c|c|c|c|c|}
\hline \\
\hline \multicolumn{7}{|c|}{$\begin{array}{l}\text { Blood/Bone Marrow } \\
\text { Haemoglobin }\end{array}$} \\
\hline Platelets & 4 & 2 & 5 & 2 & 4 & 4 \\
\hline Leukocytes & 8 & 1 & 2 & 0 & 8 & 1 \\
\hline Neutrophils & 2 & 2 & 1 & 1 & 2 & 2 \\
\hline
\end{tabular}

A) Any grade 1-2 treatment-related adverse clinical events occurring in $>10 \%$ of patients and all grade $3-4$ treatment-related adverse clinical events. B) All-cause laboratory events

(REILD), although the role of prior gemcitabine and herbal remedies prior to protocol therapy should also be considered. The patient had also received external beam radiotherapy (45 Gy in 25 fractions) to the pancreas.

\section{Treatment response and survival}

Individual best response within the liver (according to RECIST) is presented in the waterfall plot together with the tumour characteristics and CA19-9 response (Fig. 1). A PR in the liver was recorded in three patients (21\%) ( 2 confirmed; 1 unconfirmed) and stable disease (SD) in ten patients $(71 \%)$, giving a liver disease control rate of $93 \%$. CT scans of a patient with a partial response are shown in Fig. 2. Best response (at all sites) was $1 \mathrm{PR}$ (7\%), SD in nine patients (64\%) and progression in four patients (29\%). Median reduction in CA19-9 was $72 \%$. Switching to gemcitabine did not appear to contribute to the initial response.
The median liver PFS was 5.2 months (range 1.417.7 months), which exceeded the pre-specified threshold for clinical significance, and 4.4 months (range 1.416.3 months) at any site (Fig. 3). The median time to progression in the liver was derived by per protocol follow-up scans on all patients until progression of liver metastases, including patients who had progressed outside the liver as the first site of progression and may have commenced a second line of chemotherapy. PFS at any site was shorter in patients with advanced primary tumour in situ compared with those who had their primary tumour resected (median 3.4 vs. 7.8 months; $p=0.017$; Fig. 4). Two patients with liver-only metastases and a resected primary had an overall PFS of 16.3 and 7.0 months, respectively.

The first site of progression is listed in Table 3. The liver was the first site of progression in two patients: one with new lesions at 6.5 months and one with progression of

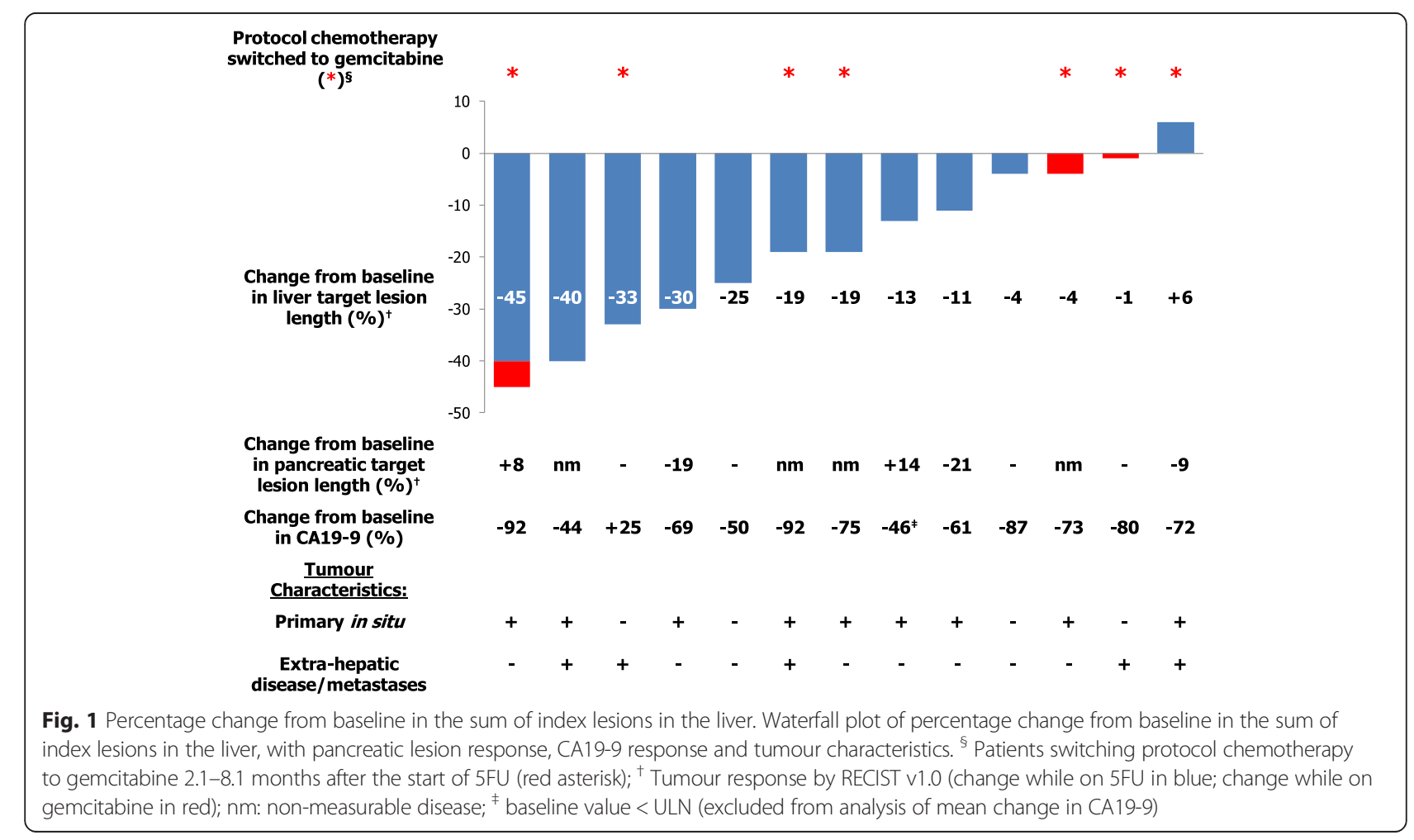



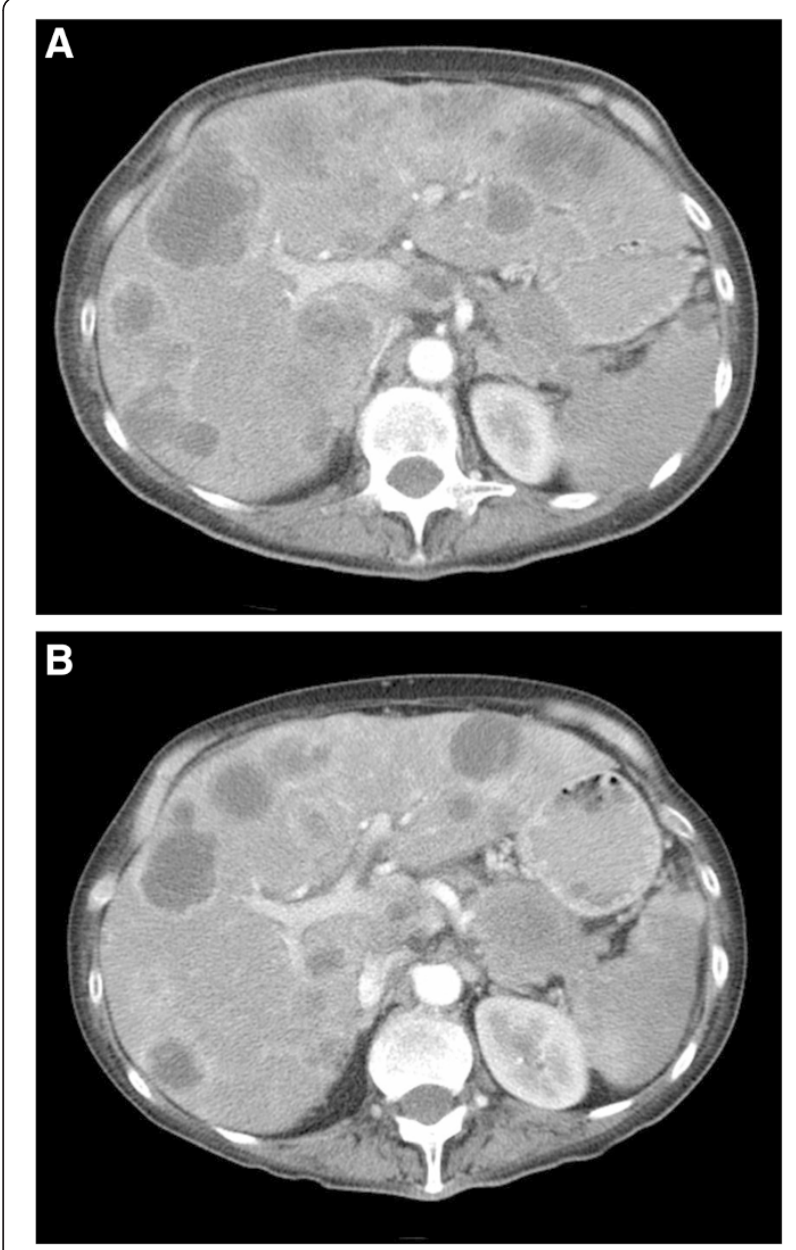

Fig. 2 Tumour response in a patient with liver-only metastases from primary pancreatic adenocarcinoma. a Contrast-enhanced CT scan prior to SIRT + 5FU. b) Follow-up contrast-enhanced $C T$ scan 3 months post-SIRT + 5FU, and prior to gemcitabine, demonstrates a partial response (40\% reduction in hepatic tumour burden), as assessed according to RECIST V1.0

existing liver lesions at 8.5 months after enrolment. Three patients $(21 \%)$ deteriorated clinically without documented evidence of disease progression. Two patients $(13 \%)$ were withdrawn from study treatment due to adverse events: one with grade 2 nausea/vomiting 3.6 months after study entry and 1 week after commencing gemcitabine, and the other with obstructive jaundice and ascites, at 2.7 months post-enrolment and 2 weeks after switching to gemcitabine.

Median OS was 5.5 months (range 1.4-19.5 months) for the entire cohort (Fig. 5) and 12.2 months (range 7.0-17.7 months) for patients with disease confined to the liver. Unplanned subgroup analysis revealed significantly longer survival in patients whose primary tumour had been resected $(n=4$; median 13.6 vs. 4.2 months; $p=0.015$; Fig. 6). For patients with liveronly metastases $(n=8)$, median survival was 6.6 months (range 3.6-17.7 months; Fig. 7). Where patients had EHD $(n=6)$, the median survival was 4.6 months (range 1.4-19.5 months). A summary of the characteristics of patients alive after 12 months is shown in Table 4.

\section{Discussion}

Metastatic pancreatic cancer carries a very poor prognosis. Progress has been frustratingly slow with numerous agents in combination with gemcitabine demonstrating promise in phase II studies, but minimal impact on OS in subsequent phase III randomised trials. Limited benefit has been demonstrated with the addition of oxaliplatin [26], cisplatin [27], capecitabine [28, 29], cetuximab [30], bevacizumab [31] or erlotinib [32] to gemcitabine. A recent study demonstrated a significant improvement in OS with FOLFIRINOX, but uncertainty remains as to whether this can be safely achieved in routine clinical practice [5].

In this first prospective study of the safety and efficacy of SIRT in advanced pancreatic cancer, 5FU rather than gemcitabine was administered concomitantly with ${ }^{90} \mathrm{Y}$ resin microspheres, thereby circumventing any potential adverse events associated with gemcitabine radiosensitisation of non-target tissue. Overall, the spectrum of adverse events in this study associated with SIRT (characterised by mild-to-moderate abdominal pain, nausea, and transient changes in liver function) and 5FU (neutropenia and thrombocytopenia) were similar to those reported in several previous trials in patients with liver metastases from CRC, in which this combination has been demonstrated to be safe $[15,16]$. The incidence of grade $3 / 4$ haematological toxicities (14\%) was consistent with the past experience with SIRT and 5FU [15, 16]. Patients who received gemcitabine no sooner than 8 weeks post-SIRT experienced a similar rate of adverse events as would be expected from gemcitabine therapy alone [4], without any evidence of REILD [33]; suggesting that gemcitabine can safely be given after SIRT.

In this study, one responding patient on 5FU developed signs suggestive of REILD and died at 7.0 months after treatment. REILD is defined as jaundice and ascites in the absence of tumour progression or bile duct obstruction commencing within 8 weeks of SIRT [33], so this event occurred well outside that window. Both rising bilirubin levels (from grade 2 on day 39 to grade 4 on day 102) and elevated alkaline phosphatase levels (which were greater than grade 1 from day 116 onwards) are recognised hallmarks of REILD [33]; although the clinical picture in this patient was complicated by use of herbal remedies [34] and gemcitabine pre-SIRT, which may have contributed to liver failure in this case as may have disease progression. The sudden death observed in one other patient was considered unlikely to be related to SIRT as sudden deaths on SIRT have not previously been reported, and the only 


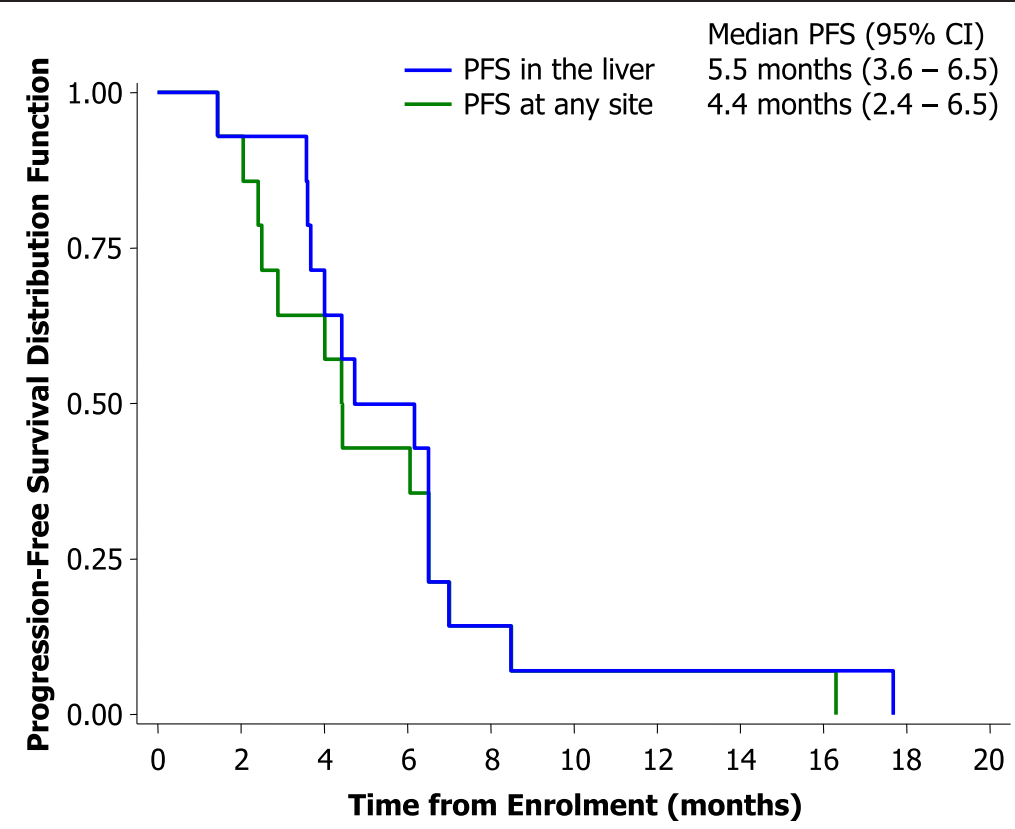

Fig. 3 Kaplan-Meier analysis of PFS and OS. 3) PFS in the liver and at any site. 4) PFS at any site stratified by the presence or absence of the primary tumour in situ. 5) OS. 6) OS stratified by the presence or absence of the primary tumour in situ. 7) OS stratified by the presence of liver-only metastases or liver plus EHD

likely cause of treatment related death (liver failure) is usually of slow onset with clinical and laboratory signs evident well in advance of patients dying.

Tumour response within the liver was encouraging, with $21 \%$ of patients (three out of 14) achieving a confirmed or unconfirmed PR and $71 \%$ (ten out of 14) achieving SD, by RECIST criteria, for a disease control rate of $93 \%$. As shown in Fig. 1, the size of the liver lesions diminished in all but one of the 13 patients who had post-SIRT imaging at 8 weeks intervals and no patient had progressive disease within the liver on initial follow-up CT imaging. With limited radiological response on gemcitabine, it would seem reasonable to conclude that the recorded response was

Table 3 Site of first progression $(n=14)$

\begin{tabular}{lll}
\hline Location of first progression & Patients & \\
\cline { 2 - 3 } & Number & Percentage \\
\hline Documented progression on CT & 2 & 20 \\
Liver & 2 & 13 \\
Lung & 1 & 7 \\
Lymph node & 1 & 7 \\
Peritoneum & 1 & 7 \\
Pleural effusion & & \\
No progression documented & 3 & 20 \\
Clinical deterioration & 2 & 13 \\
Death without progression & 2 & 13 \\
Withdrawn due to adverse events & & \\
\hline
\end{tabular}

largely due to the protocol treatment rather than subsequent gemcitabine.

The imaging results are also corroborated by the decline in CA19-9 observed in 12 of 13 patients with an elevated CA19-9 at baseline, including all but one of those with EHD. Similar to the experience in SIRTtreated CRC liver metastases $[35,36]$, the decline in tumour marker was rapid and appeared to predict later CT response and PFS.

The results of our phase II study compare favourably to a time to progression in the randomised controlled trial by Burris et al. of 0.9 months for 5FU alone, and 2.3 months for gemcitabine and response rate with $5 \mathrm{FU}$ alone of 0 and $19 \%$ SD. Subsequently Cunningham et al. has recorded small incremental improvements in PFS with gemcitabine combined with capecitabine compared with gemcitabine alone (5.3 vs. 3.8 months), which have been accompanied by a small survival benefit (6.2 vs. 7.1 months) [29]. The median PFS reported for patients treated with FOLFIRINOX in the recent randomised study was 6.4 months [5]. Significantly however, the studies by Cunningham et al. [28] and that of Burris et al. [4] included many patients with locally advanced disease (29 and $26 \%$ of patients, respectively), for whom the median PFS would be expected to be superior, whereas the current study and the FOLFIRINOX study only included patients with metastatic disease.

As expected for a liver-directed therapy, studies of SIRT in patients with CRC have demonstrated better outcomes in patients with disease confined to the liver [18, 37]. In 


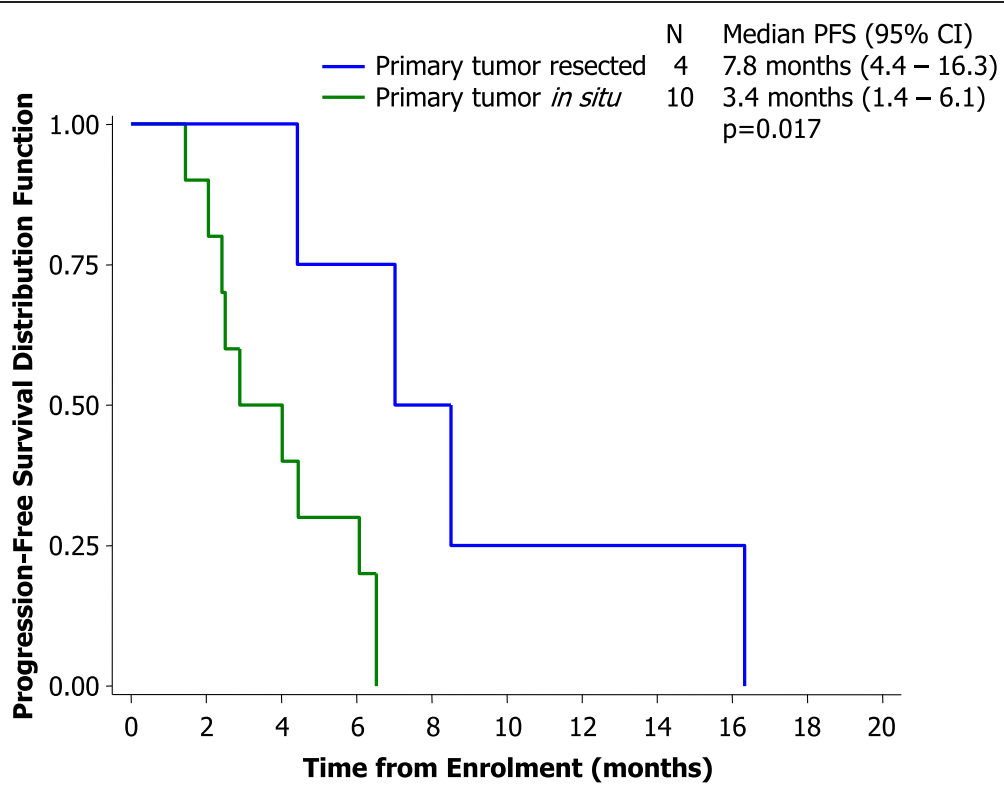

Fig. 4 Kaplan-Meier analysis of PFS and OS

the current trial, outcomes likewise appeared related to extent of disease outside the liver, with the best results seen in the two patients with liver-only disease (OS of 7.0 and 17.7 months) and the worst outcomes in the four patients with an intact primary and liver metastases plus EHD (median OS of 4.2 months). These results suggest that further studies of SIRT in pancreatic cancer liver metastases should be confined to the population of patients with liver-only disease who have had their primary lesion resected or who have well-controlled primary disease. These analyses should not be used to select patients for treatment outside of clinical trials, as SIRT remains an experimental treatment option in this disease type.

\section{Conclusions}

The data obtained from this study of the combination of SIRT and 5FU in the treatment of liver metastases from primary pancreatic cancer demonstrated evidence of effective

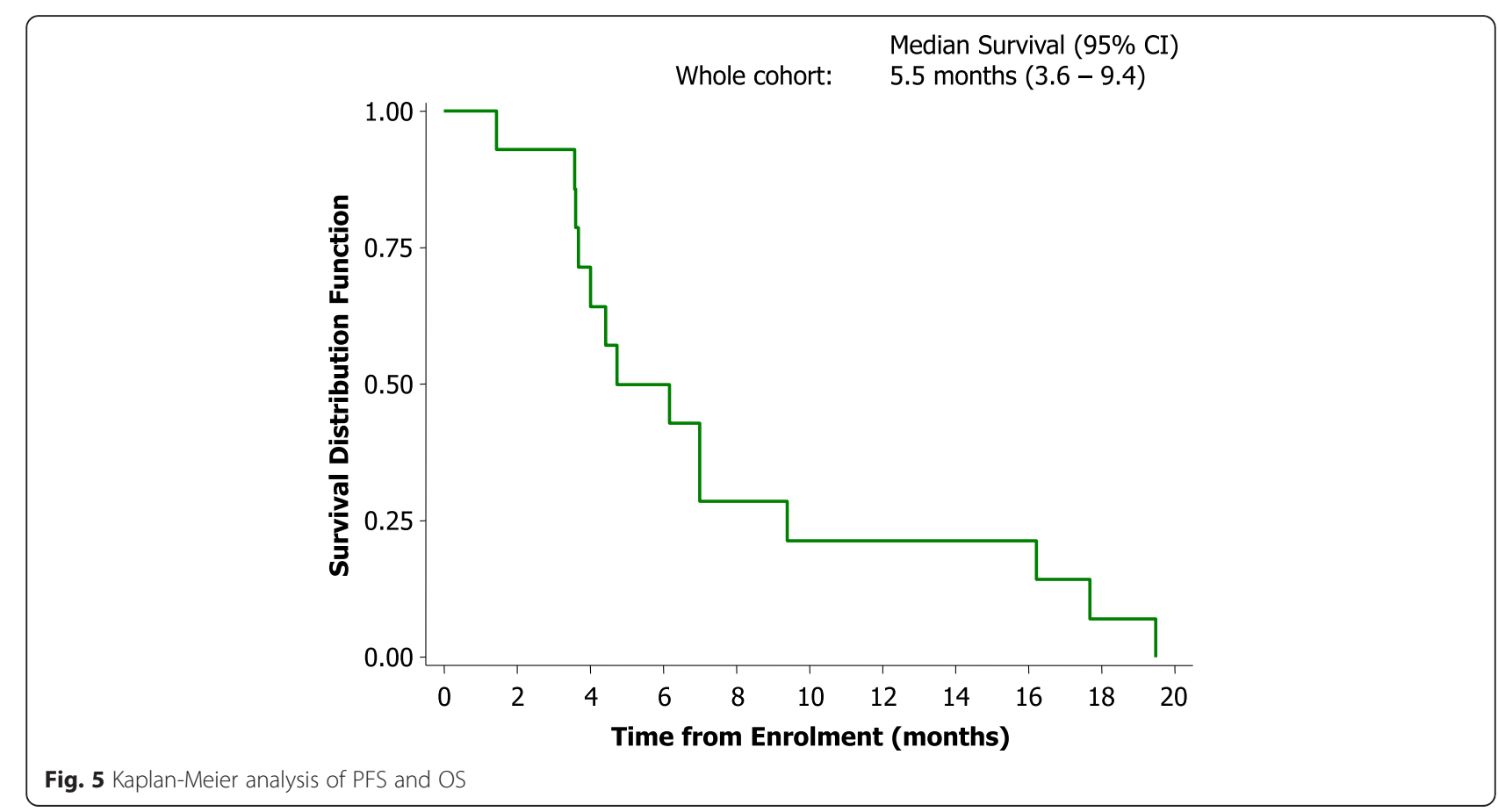




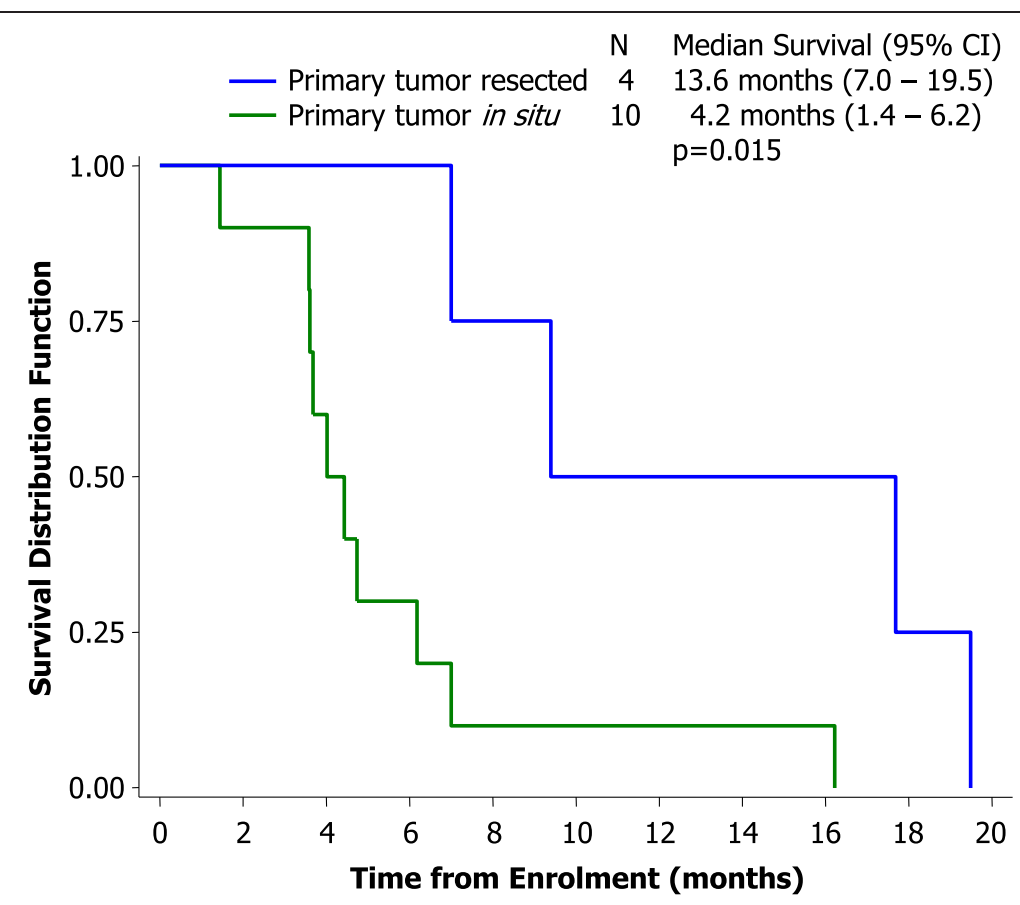

Fig. 6 Kaplan-Meier analysis of PFS and OS

disease control of liver metastases from pancreatic adenocarcinoma, with a disease control rate of $93 \%$ and a liver PFS of 5.2 months. However, the combination of SIRT and 5FU resulted in a toxicity profile that was significant and the safety of this approach in patients with metastatic pancreatic cancer will need to be confirmed in subsequent studies. This combination of therapy is likely to be of most benefit in selected patients with a resected primary tumour and liver only disease. Ultimately though, randomised trials will be needed to prove the role of SIRT in combination with chemotherapy in metastatic pancreatic cancer, and to define the patients who will most benefit from this treatment. Strategies combining SIRT with gemcitabine are likely to be limited by the doses of gemcitabine that could be given safely with SIRT, without compromising its systemic activity. Several studies in CRC have demonstrated that SIRT

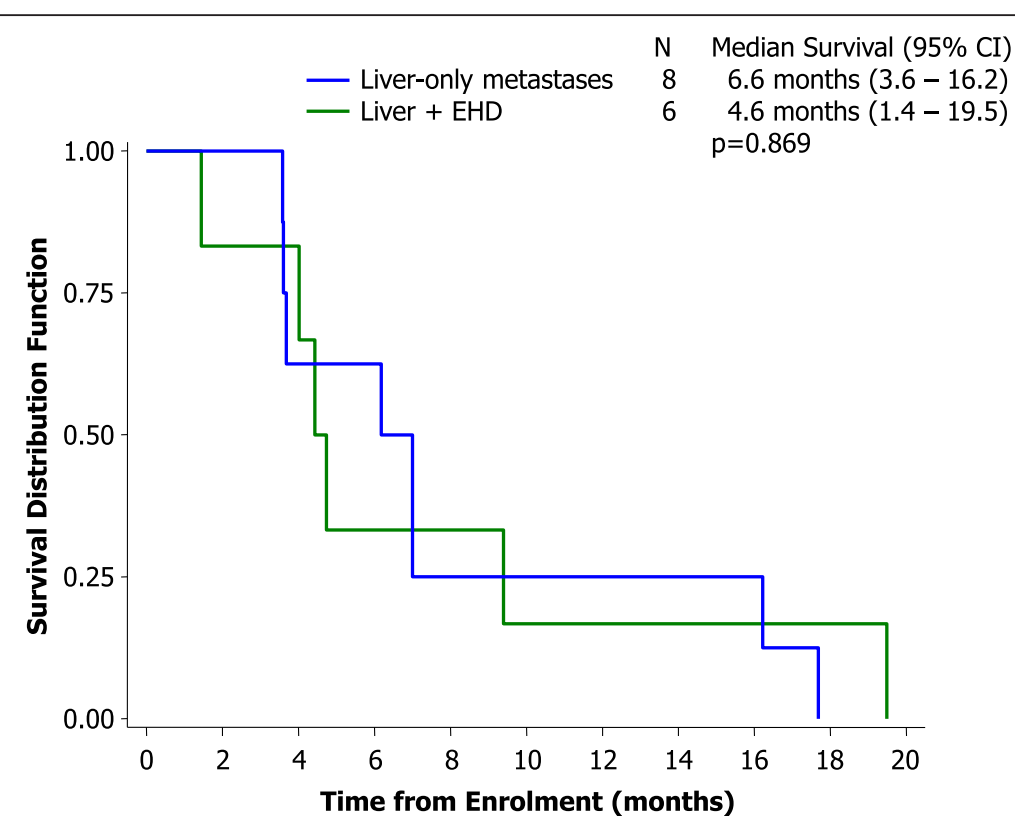

Fig. 7 Kaplan-Meier analysis of PFS and OS 
Table 4 Characteristics of patients who survived greater than 12 months

\begin{tabular}{llllllll}
\hline Patient & $\begin{array}{l}\text { Stage at } \\
\text { diagnosis }^{\mathrm{a}}\end{array}$ & $\begin{array}{l}\text { Status of primary } \\
\text { tumour }\end{array}$ & Site of metastases & $\begin{array}{l}\text { PFS in the liver } \\
\text { (months) }\end{array}$ & $\begin{array}{l}\text { PFS } \\
\text { (months) }\end{array}$ & $\begin{array}{l}\text { Survival } \\
\text { (months) }\end{array}$ & $\begin{array}{l}\text { Site of first disease } \\
\text { progression }\end{array}$ \\
\hline Patient 1 & Tx & Primary in situ & Liver only & 6.5 & 6.5 & 16.2 & Liver \\
Patient 2 & T2 & Prior resection & Liver only & 17.7 & 16.3 & 17.7 & Pleural effusion \\
Patient 3 & T3 & Prior resection & Liver and lymph nodes & 8.5 & 8.5 & 19.5 & Liver \\
\hline
\end{tabular}

${ }^{\mathrm{a}}$ According to United Network for Organ Sharing staging criteria

can be safely combined with FOLFOX [15-17] and with irinotecan [38], suggesting that initial cycles with FOLFOX or irinotecan might be an attractive strategy.

\section{Human research and ethics committees approval}

This study was approved by the ethics committees of Melbourne Health, Parkville, Australia (for patients enrolled at Western Hospital) and Mount Hospital, Perth, Australia (for patients enrolled at Mount Hospital)

\section{Additional file}

\section{Additional file 1: Dosimetry tables for ${ }^{90} \mathrm{Y}$-resin microspheres. The} following tables (Table S1A to S1C) provided by Sirtex Medical Limited, Sydney, Australia were used in this phase II study to guide the dosimetry for ${ }^{90} Y$-resin microspheres (SIR-Spheres). The tables, based a modification of the Body Surface Area (BSA) formula, calculated the activity in gigabecquerels $(\mathrm{GBq})$ of ${ }^{90} \mathrm{Y}$ which was to be implanted determined from the BSA and percentage tumour involvement of whole liver for each patient. Three tables were provided so that the implanted activity of ${ }^{90} \mathrm{Y}$ could be further adjusted for each patient according to extent of lung shunting (0-10\%; 11-15\%; 16-20\%). The tables provide a more user-friendly method for calculating the dosimetry for ${ }^{90}$ Y-resin microspheres, while at the same including slight modifications to the dosing, in order to improve the safety of this procedure in patients with either a very low $(<10 \%)$ or high (>60\%) tumour volume in the liver. (DOCX $43 \mathrm{~kb})$

\section{Abbreviations}

5FU: 5-fluorouracil; Y: Yttrium-90; CA19-9: Cancer antigen 19-9; CRC: Colorectal cancer; CT: Computed tomography; EHD: Extrahepatic disease/metastases; FOLFIRINOX: Folinic acid, fluorouracil, irinotecan, oxaliplatin; FOLFOX: Folinic acid, fluorouracil, oxaliplatin; mCRC: Metastatic colorectal cancer; MRI: Magnetic resonance imaging; NCI-CTCAE: National Cancer Institute Common Terminology Criteria for Adverse Events; OS: Overall survival; PFS: Progression-free survival; PR: Partial response; RECIST: Response Evaluation Criteria In Solid Tumours;

REILD: Radioembolization-induced liver disease; SD: Stable disease; SIRT: Selective internal radiation therapy; ULN: Upper limit of normal; WHO: World Health Organization.

\section{Competing interests}

PG and GVH have received honoraria from SIRTEX for participation in advisory boards and for presentations. DC and MT are employees of Sirtex Medical Limited. The remaining authors declare that they have no competing interests.

\section{Authors' contributions}

PG conceived of the study, and participated in its design and co-ordination and drafted the manuscript. CD assisted in analysis of data and in drafting the manuscript. LL recruited patients to the study and reviewed the manuscript, DC assisted in study design, analysis and manuscript preparation. MT assisted in study design, analysis and manuscript preparation, DP was involved in delivery of study treatment and reviewed the manuscript, GB was involved in delivery of study treatment and reviewed the manuscript, RD was involved in delivery of study treatment and reviewed the manuscript, ML was involved in delivery of study treatment and reviewed the manuscript, GVH participated in study design, recruited patients and reviewed the manuscript.

\section{Acknowledgements}

This study was supported by SIRTEX Medical. The authors would like to thank Rae Hobbs for her help in editing this manuscript, funding for which was provided by Sirtex Medical Ltd.

\section{Author details}

'Department of Medical Oncology, Royal Melbourne Hospital, Grattan Street, Parkville, VIC 3050, Australia. ${ }^{2}$ Department of Medical Oncology, Western Hospital, Melbourne, Australia. ${ }^{3}$ Sirtex Medical Limited, Sydney, Australia. ${ }^{4}$ Perth Radiological Clinic, Mount Hospital, Perth, Australia. ${ }^{5}$ Mount Nuclear Medicine, Mount Hospital, Perth, Australia. ${ }^{6}$ Department of Radiology, Royal Melbourne Hospital, Melbourne, Australia. ${ }^{7}$ Department of Nuclear Medicine, Royal Melbourne Hospital, Melbourne, Australia. ${ }^{8}$ Perth Oncology, Mount Hospital, Perth, Australia.

Received: 25 February 2014 Accepted: 16 October 2015 Published online: 26 October 2015

\section{References}

1. Pancreatic Action Support Network: the alarming rise of pancreatic cancer deaths in the United States: why we need to stem the tide. http:// www.pancan.org/section_research/reports/pdf/incidence_report_2012.pdf.

2. World health Organization. Estimated cancer incidence, mortality, prevalence and disability-adjusted life years (DALYs) worldwide in 2008. GLOBOCAN (International Agency for Research on Cancer). 2008. http:// globocan.iarc.fr/.

3. Jemal A, Siegel R, Xu J, Ward E. Cancer statistics, 2010. CA Cancer J Clin. 2010;60:277-300.

4. Burris HA, Moore MJ, Andersen J, Green MR, Rothenberg ML, Modiano MR, et al. Improvements in survival and clinical benefit with gemcitabine as first line therapy for patients with advanced pancreas cancer. J Clin Oncol. 1997;15:2403-13.

5. Conroy T, Desseigne F, Ychou M, Bouché O, Guimbaud R, Bécouarn Y, et al. FOLFIRINOX versus gemcitabine for metastatic pancreatic cancer. $N$ Engl J Med. 2011;364:1817-25.

6. Lorgis V, Chauffert B, Gentil J, Ghiringhelli F. Influence of localization of primary tumor on effectiveness of 5-Fluorouraci/leucovorin combined with irinotecan and oxaliplatin (FOLFIRINOX) in patients with metastatic pancreatic adenocarcinoma: a retrospective study. Anticancer Res. 2012;32:4125-30.

7. Hosein PJ, Macintyre J, Kawamura C, Maldonado JC, Ernani V, Loaiza-Bonilla A, et al. A retrospective study of neoadjuvant FOLFIRINOX in unresectable or borderline-resectable locally advanced pancreatic adenocarcinoma. BMC Cancer. 2012;12:199.

8. Peddi PF, Lubner S, McWilliams R, Tan BR, Picus J, Sorscher SM, et al. Multi-institutional experience with FOLFIRINOX in pancreatic adenocarcinoma. JOP. 2012;13:497-501.

9. Evans DB, Abbruzzese JL Willett CG. Cancer of the pancreas. In: DeVita Jr VT, Hellman S, Rosenberg SA, editors. Cancer: principles and practice of oncology. 6th ed. Philadelphia: Lippincott Williams \& Wilkins; 2001. p. 1126-61.

10. Sangro B, Carpanese L, Cianni R, Golfieri R, Gasparini D, Ezziddin S, et al. Survival after yttrium-90 resin microsphere radioembolization of hepatocellular carcinoma across Barcelona clinic liver cancer stages: a European evaluation. Hepatology. 2011;54:868-78.

11. Salem R, Lewandowski RJ, Mulcahy MF, Riaz A, Ryu RK, Ibrahim S, et al. Radioembolization for hepatocellular carcinoma using yttrium-90 microspheres: a comprehensive report of long-term outcomes. Gastroenterology. 2010;138:52-64.

12. Sangro B, Iñarrairaegui M, Bilbao Jl. Radioembolisation for hepatocellular carcinoma. J Hepatol. 2012;56:464-73.

13. Kennedy AS, Dezarn WA, McNeillie P, Coldwell D, Nutting C, Carter D, et al. Radioembolization for unresectable neuroendocrine hepatic metastases 
using resin ${ }^{90} \mathrm{Y}$-microspheres: early results in 148 patients. Am J Clin Oncol. 2008; $31: 271-9$

14. King J, Quinn R, Glenn DM, Janssen J, Tong D, Liaw W, et al. Radioembolization with selective internal radiation microspheres for neuroendocrine liver metastases. Cancer. 2008;113:921-9.

15. Hendlisz A, Van den Eynde M, Peeters M, Maleux G, Lambert B, Vannoote J et al. Phase III trial comparing protracted intravenous fluorouracil infusion alone or with yttrium-90 resin microspheres radioembolization for liver-limited metastatic colorectal cancer refractory to standard chemotherapy. J Clin Oncol. 2010;28:3687-94.

16. Van Hazel G, Blackwell A, Anderson J, Price D, Moroz P, Bower G, et al. Randomised phase 2 trial of SIR-Spheres plus fluorouracil/leucovorin chemotherapy versus fluorouracil/leucovorin chemotherapy alone in advanced colorectal cancer. J Surg Oncol. 2004;88:78-85.

17. Kosmider S, Tan TH, Yip D, Dowling R, Lichtenstein M, Gibbs P. Radioembolization in combination with systemic chemotherapy as first-line therapy for liver metastases from colorectal cancer. J Vasc Interv Radiol. 2011;22:780-6.

18. Sharma RA, Van Hazel GA, Morgan B, Berry DP, Blanshard K, Price D, et al. Radioembolization of liver metastases from colorectal cancer using yttrium90 microspheres with concomitant systemic oxaliplatin, fluorouracil, and leucovorin chemotherapy. J Clin Oncol. 2007;25:1099-106.

19. Coldwell DM, Kennedy AS, Nutting CW. Use of yttrium-90 microspheres in the treatment of unresectable hepatic metastases from breast cancer. Int J Radiat Oncol Biol Phys. 2007;69:800-4.

20. Jakobs TF, Hoffmann RT, Fischer T, Stemmler HJ, Tatsch K, La Fougere C, et al. Radioembolization in patients with hepatic metastases from breast cancer. J Vasc Interv Radiol. 2008;19:683-90.

21. Bangash AK, Atassi B, Kaklamani V, Rhee TK, Yu M, Lewandowski RJ, et al. ${ }^{90}$ Y radioembolization of metastatic breast cancer to the liver: toxicity, imaging response, survival. J Vasc Interv Radiol. 2007;18:621-8.

22. Cao C, Yan TD, Morris DL, Bester L. Radioembolization with yttrium-90 microspheres for pancreatic cancer liver metastases: results from a pilot study. Tumori. 2010;96:955-8.

23. Gulec SA, Wheeler J, Pennington K, Hall M, Bruetman D, Westbrook C, et al. Chemotherapy with Yttrium-90 microsphere selective internal radiation treatment and selective external radiation treatment in patients with metastatic pancreatic cancer. J Interven Oncol. 2009;2:84-92.

24. Kornek GV, Pötter R, Selzer E, Schratter A, Ulrich-Pur H, Rogy M, et al. Combined radiochemotherapy of locally advanced unresectable pancreatic adenocarcinoma with mitomycin C plus 24-hour continuous infusional gemcitabine. Int J Radiat Oncol Biol Phys. 2001;49:665-71.

25. de Lange SM, van Groeningen CJ, Meijer OW, Cuesta MA, Langendijk JA, van Riel JM, et al. Gemcitabine-radiotherapy in patients with locally advanced pancreatic cancer. Eur J Cancer. 2002;38:1212-7.

26. Louvet C, Labianca R, Hammel P, Lledo G, Zampino MG, André T, et al. Gemcitabine in combination with oxaliplatin compared with gemcitabine alone in locally advanced or metastatic pancreatic cancer: results of a GERCOR and GISCAD phase III trial. J Clin Oncol. 2005;23:3509-16.

27. Colucci G, Labianca R, Di Costanzo F, Gebbia V, Cartenì G, Massidda B, et al. Randomized phase III trial of gemcitabine plus cisplatin compared with single-agent gemcitabine as first-line treatment of patients with advanced pancreatic cancer: the GIP-1 study. J Clin Oncol. 2010;28:1645-51.

28. Herrmann R, Bodoky G, Ruhstaller T, Glimelius B, Bajetta E, Schüller J, et al. Gemcitabine plus capecitabine compared with gemcitabine alone in advanced pancreatic cancer: a randomized, multicenter, phase III trial of the Swiss Group for Clinical Cancer Research and the Central European Cooperative Oncology Group. J Clin Oncol. 2007;25:2212-7.

29. Cunningham D, Chau I, Stocken DD, Valle JW, Smith D, Steward W, et al. Phase III randomized comparison of gemcitabine versus gemcitabine plus capecitabine in patients with advanced pancreatic cancer. J Clin Oncol. 2009:27:5513-8.

30. Philip PA, Benedetti J, Corless CL, Wong R, O'Reilly EM, Flynn PJ, et al. Phase III study comparing gemcitabine plus cetuximab versus gemcitabine in patients with advanced pancreatic adenocarcinoma: Southwest Oncology Group-directed intergroup trial S0205. J Clin Oncol. 2010;28:3605-10.

31. Kindler HL, Niedzwiecki D, Hollis D, Sutherland S, Schrag D, Hurwitz H, et al. Gemcitabine plus bevacizumab compared with gemcitabine plus placebo in patients with advanced pancreatic cancer: phase III trial of the Cancer and Leukemia Group B (CALGB 80303). J Clin Oncol. 2010;28:3617-22.
32. Moore MJ, Goldstein D, Hamm J, Figer A, Hecht JR, Gallinger S, et al. Erlotinib plus gemcitabine compared with gemcitabine alone in patients with advanced pancreatic cancer: a phase III trial of the National Cancer Institute of Canada Clinical Trials Group. J Clin Oncol. 2007:25:1960-6.

33. Sangro B, Gil-Alzugaray B, Rodriguez J, Sola I, Martinez-Cuesta A, Viudez A, et al. Liver disease induced by radioembolization of liver tumors: description and possible risk factors. Cancer. 2008;112:1538-46.

34. Whiting PW, Clouston A, Kerlin P. Black cohosh and other herbal remedies associated with acute hepatitis. MJA. 2002;177:432-5.

35. Jakobs TF, Hoffmann RT, Dehm K, Trumm C, Stemmler HJ, Tatsch K, et al. Hepatic yttrium-90 radioembolization of chemotherapy-refractory colorectal cancer liver metastases. J Vasc Interv Radiol. 2008;19:1187-95.

36. Gray BN, Burton MA, Kelleher DK, Anderson J, Klemp P. Selective internal radiation (SIR) therapy for treatment of liver metastases: measurement of response rate. J Surg Oncol. 1989;42:192-6.

37. Nace GW, Steel JL, Amesur N, Zajko A, Nastasi BE, Joyce J, et al. Yttrium-90 radioembolization for colorectal cancer liver metastases: a single institution experience. Int J Surg Oncol. 2011;2011:571261.

38. van Hazel GA, Pavlakis N, Goldstein D, Olver IN, Tapner MJ, Price D, et al. Treatment of fluorouracil-refractory patients with liver metastases from colorectal cancer by using Yttrium-90 resin microspheres plus concomitant systemic irinotecan chemotherapy. J Clin Oncol. 2009;27:4089-95.

\section{Submit your next manuscript to BioMed Central and take full advantage of:}

- Convenient online submission

- Thorough peer review

- No space constraints or color figure charges

- Immediate publication on acceptance

- Inclusion in PubMed, CAS, Scopus and Google Scholar

- Research which is freely available for redistribution 